\title{
Six degrees of freedom arc welding robot off-line programming based on MATLAB
}

\author{
Zimu $\mathrm{Li}^{1, \mathrm{a}}$, Xinyu Wang ${ }^{2, \mathrm{~b}}$ and Wenming Zhang ${ }^{3, \mathrm{c}^{*}}$ \\ ${ }^{1}$ Tiandi Benniu Yinqi Equipment Co. Ltd. of Ningxia, China \\ ${ }^{2}$ Shenyang University, China \\ ${ }^{3}$ Shenyang University, China

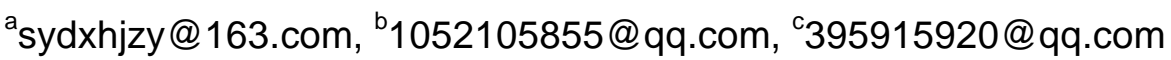

\begin{abstract}
Keywords: Arc welding robot; MATLAB; Off-line programming
Abstract. With the constant improvement of the level of industrial automation, robots are increasingly used in various fields of industrial production which is one of the most widely used welding robot. Today, however, people mainly adopt teaching methods to control the robot manually, it has some shortcomings, such as low efficiency, poor accuracy and time consuming. To solve this problem, this paper proposes an off-line programming method based on MATLAB software. Robot moving trajectories are generated by the MATLAB software, and carry on simulation, make the robot automatically, moving along the desired trajectory welding task. Reduce the work intensity of the workers and improve the productivity.
\end{abstract}

\section{Introduction}

Nowadays, robot technology rapid development, people expect the robot can replace man to finish complex work in a dangerous environment. Industrial robots have been widely used in all walks of life, with the rapid development of manufacturing industry, the development of industrial automation has become the theme. The traditional manual teaching robots has been increasingly cannot meet the development needs of today. On the one hand, the workload of manual teaching is very heavy, on the other hand, it require the operator observe points by the naked eye, so it will produce large error, urgently need to use the off-line programming method for processing. This article will use the MATLAB software $3 \mathrm{~d}$ modeling is combined with SRVWS off-line programming software developed by SIASUN company and robot simulation function, to research the complex saddle weld off-line programming technology.

\section{Coordinate system instructions ${ }^{[1]}$}

In order to accurately control and describe the motion of the robot, we want to establish multiple robot coordinate system. Making robots can move accurately based on the coordinate system. This article involves the base coordinate system, the wrist system, and the tools coordinate system. Next these coordinates are introduced in detail.

The base coordinate system $\{B\}$

The base coordinate system $\{B\}$ is located in the center of the base.

The wrist coordinate system $\{\mathrm{W}\}$

The wrist coordinate system $\{\mathrm{W}\}$ attached to the end of the connecting rod manipulator, in most cases, the origin point of wrist coordinate $\{\mathrm{W}\}$ in manipulator wrist, it moved along with the end of the connecting rod manipulator.

\section{The tool coordinate system $\{\mathbf{T}\}$}

The tool coordinate system $\{\mathrm{T}\}$ attached to the robot by the end of the gripping tool, when the hand without clamping tools, the origin point of the tool coordinate system $\{\mathrm{T}\}$ lies between the robot's fingers. 


\section{The calibration of the tool coordinate system ${ }^{[2]}$}

We will weld a saddle trajectory at this time, so the welding torch should be mounted in the robot wrist. Robot tool coordinate system origin point should be placed at the end of the clamping electrode holder not the wrist of the robot. This needs us to calibrate the tool coordinate system, make good robot end actuators based on calibration tool coordinate system movement, to ensure its trajectory accuracy and precision.

Calibration tool coordinate system, we adopt five calibration method. Namely: find fixed point (which is best for the vertical profile of sharp point) near the robot, make the tool center point close to that point, which is infinitely close, don't touch. After recording the current position of the robot. The shaft of the mobile robot, the change of welding torch posture, the tool center point from the other direction close to the fixed point, and record the current position of the robot. Records a total of five times, the robot will automatically generate the parameters of the tool coordinate system.

After the parameters of the tool coordinate system automatically generated, under the tools or rectangular coordinate system, transformation of welding torch posture, if the tool center point basically remain unchanged, prove the generated parameters of the tool coordinate system is correct. As is shown in Fig.1.

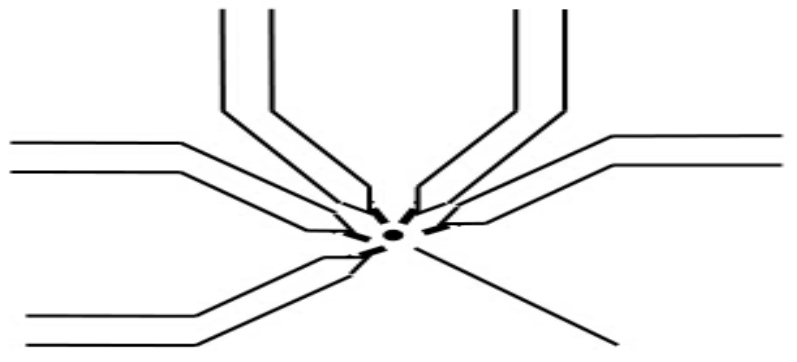

Fig.1. Five calibration method

\section{Coordinate system transformation}

We simulate a saddle trajectory use the method of creating the coordinate points. Coordinate point is created based on the base coordinate system. But considering the accessibility to satisfy the robot movement, collision during exercise, produce dead point, singular point, etc., we need to change the coordinate systems. First, we find the welding workpieces by using the method of manual operation of saddle trajectory space origin of coordinates, to build a coordinate system for the origin point $\{\mathrm{A}\}$, and record the coordinates. And then make the base coordinate system $\{\mathrm{B}\}$ through translation, rotation transformation to the coordinate $\{\mathrm{A}\}$.

Since the position and posture of the two coordinate system are not identical. So make the based coordinate system $\{\mathrm{B}\}$ rotate to same gesture to the coordinate system $\{\mathrm{A}\}$, and then translate a distance, make the two coordinate system origin point at the same point, then complete the coordinate system transformation.

$$
{ }^{A} P={ }^{A} R{ }^{B} P+{ }^{A} P_{B}
$$

\section{Generation of saddle trajectory}

The welding trajectory formed by two tubes, a great inner diameter is $213 \mathrm{~mm}$, another tube diameter is $96 \mathrm{~mm}$. The two root canal are the curve form of weld, generating the weld path through the MATLAB software. First, enter the following statement in "Window" Command.

- $\mathrm{t}=0: \mathrm{pi} / 30: 6^{*} \mathrm{pi}$;

- $\mathrm{x}=1000 * \cos (\mathrm{t})$;

- $\mathrm{y}=1000 * \sin (\mathrm{t})$;

- $\quad \mathrm{z}=\operatorname{sqrt}\left(45369-9216^{*} \sin (\mathrm{t}){ }^{*} \sin (\mathrm{t})\right)$;

- $\operatorname{plot} 3(\mathrm{x}, \mathrm{y}, \mathrm{z})$; 
After clicking "enter" button, the software will automatically generate a saddle trajectory. On generating saddle trajectory, we need to use "Data Cursor" select enough points, and record the coordinates of the points, as shown in Fig.2.

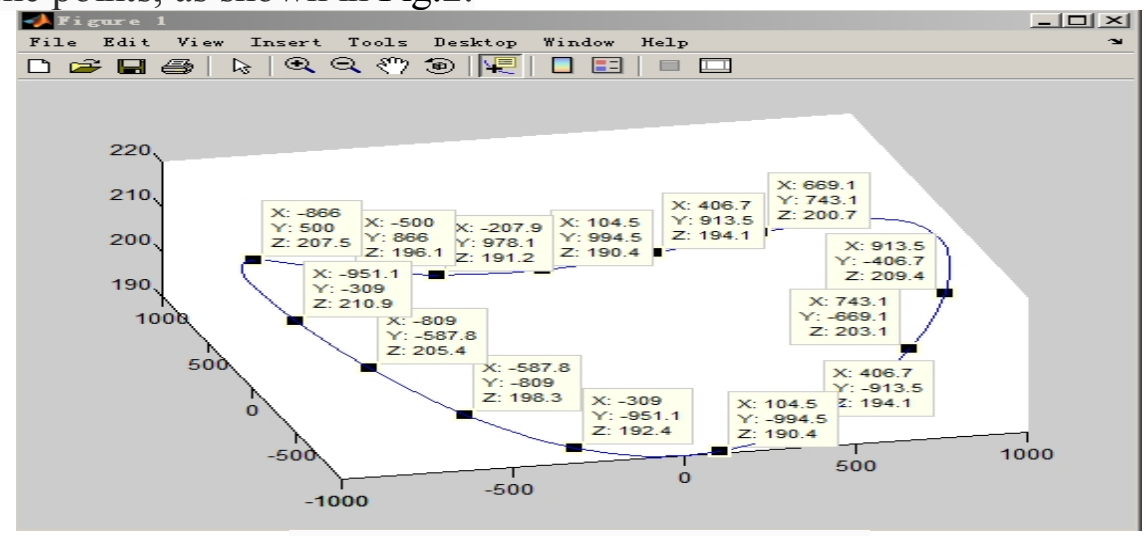

Fig.2.Saddle trajectory coordinates

\section{Off-line programming and simulation in SRVWS software ${ }^{[3]}$}

Before the off-line programming and simulation, we need a series of preparations. First of all, using the five point method to calibrate the tool coordinate system, recording the teach box of coordinate system of the position and posture. Then, adopting manual teaching method to find the welding workpieces of saddle trajectory space coordinate origin point, recording the teach box in the same coordinate position and posture. Then we will use MATLAB software programming, to simulate a three-dimensional space saddle trajectory. In the use of MATLAB functions, three dimensional saddle trajectory in the space on the selection of uniform, if circumstances permit, you can pick and choose the point as much as possible of the robot trajectory, the accuracy will be improved. Then record the selected points of the coordinates. Finally, we will translate and rotate the coordinate system, calculate the Coordinate values after changed of the selected point and recorded, for the use in SRVWS software created in the target. After all preparations were made, we take saddle trajectory operations for example, off-line programming and simulation.

Step 1: open the SRVWS software, building a new workstation, select the third module of existing templates rh10-2.Then set the workstation, authors, the location of the name, it created a homework.

Step 2: calibration tool coordinate system. Click on the "virtual trial teaching" $\rightarrow$ "tool coordinate system", we will input Coordinate values with five point calibration, click "create", completes the tool coordinate system calibration.

Step 3: create the target point. Click on the "virtual trial teaching" to "target" $\rightarrow$ "create the target", open the create the target dialog box. We have been well calculated after the transformation of selected point coordinates, the rotation Angle input to the dialog box, click the "add", add a target, then in turn each target repeat the above operation. Then click "create", complete the creation of a target. We can observe the space composed of various target three dimensional saddle trajectory work in $3 \mathrm{~d}$ space, Finally right click on the location point, click on the "tools" $\rightarrow$ new calibration tool coordinate system. Tools at the center of the store and the saddle trajectory coincides, improved the precision of the robot's movement, as shown in Fig.3

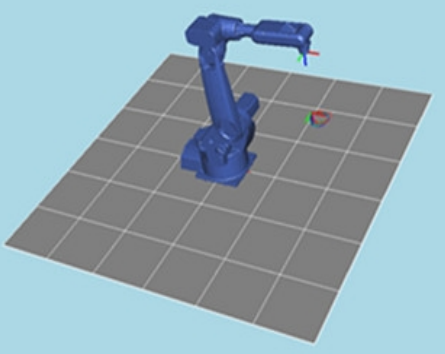

Fig.3. Saddle shaped track 
Step 4: point configuration. First in the "track sections list" to create a path, copies all the above create a good point to track sections in the list. Later, right-click on the first path points, select "configuration". General project list will appear a few configuration solutions, generally choose the configurations of the first configuration solution, choosing configuration to avoid the shaft joints close to the soft limit. Then click "apply", complete the configuration of the first point. Then right click on the track section name, click on the "automatic configuration, the configuration is successful, the icon will change. Then complete the configuration of all points.

Step 5: the simulation run. Right click on the track section name, and click "simulation", we will see a robot run automatically, in our space saddle trajectory check the track section is workable.

Step 6: download the homework. Finally, download the programming good homework to teach box, to convey instructions to the robot, so as to control the trajectory of the welding task automatically.

Robot, in general, in the whole period of orbit, smooth operation, smooth, highly motion precision , the welding seam quality can meet the requirements, more convenient than traditional manual teaching methods, greatly improve the work efficiency of the robot.

\section{Conclusions}

This article introduces the SIASUN robot off-line programming with MATLAB software research, after many experiments, the smooth movement of robot in the saddle trajectory, trajectory precision is very high, which can meet the requirement of off-line programming.

This article gives a method of robot off-line programming, the reader can has a deeper understanding to robot off-line programming. It still exist deficiencies, of course, I will read more literatures, combining previous research results to improve continuously.

Finally thanks to the teacher's guidance and the patience of my senior fellow apprentice!

\section{Literature References}

[1] JJohnJ.Craig. Introduction to Robotics:Mechanics and Control,Third Edition,China Machine press ,Beijing,2006,68-69.

[2] CSC_V2.3 SIASUN industrial robots general manual, 70-73, In Chinese

[3] SRVWS The user manual In Chinese 